\title{
Implementation of Bronchoscopic Lung Volume Reduction Using One-Way Endobronchial Valves: A Retrospective Single-Centre Cohort Study
}

\author{
Rein Posthuma ${ }^{a, b, c}$ Anouk W. Vaes ${ }^{a}$ Kim H.M. Walraven ${ }^{b, c}$ Peyman Sardari Nia ${ }^{d}$ \\ Jan U.Schreiber ${ }^{\mathrm{e}}$ Hester A. Gietema ${ }^{\mathrm{f} g}$ Geertjan Wesseling ${ }^{c}$ Emiel F.M. Wouters ${ }^{\mathrm{a}} \mathrm{h}$ \\ Lowie E.G. W. Vanfleteren ${ }^{\mathrm{i}, \mathrm{j}}$ \\ aDepartment of Research and Development, Ciro, Horn, The Netherlands; ${ }^{b}$ NUTRIM School of Nutrition and Translational \\ Research in Metabolism, Maastricht, The Netherlands; 'Department of Respiratory Medicine, Maastricht University Medical \\ Centre (MUMC+), Maastricht, The Netherlands; ${ }^{\mathrm{d} D e p a r t m e n t}$ of Cardiothoracic Surgery, Maastricht University Medical \\ Centre, Maastricht, The Netherlands; 'Department of Anaesthesiology, Maastricht University Medical Centre, Maastricht, \\ The Netherlands; 'Department of Radiology, Maastricht University Medical Centre+, Maastricht, The Netherlands; ${ }^{9} \mathrm{GROW}$ \\ School of Oncology and Developmental Biology, Maastricht, The Netherlands; 'hudwig Boltzmann Institute for Lung \\ Health, Vienna, Austria; 'Department of Respiratory Medicine and Allergology, COPD Centre, Sahlgrenska University \\ Hospital, Gothenburg, Sweden; Institute of Medicine, Department of Internal Medicine and Clinical Nutrition, Sahlgrenska \\ Academy, University of Gothenburg, Gothenburg, Sweden
}

\section{Keywords}

Bronchoscopic lung volume reduction · Intervention ·

Interventional bronchoscopy · Emphysema

\begin{abstract}
Background: Bronchoscopic lung volume reduction (BLVR) using 1-way endobronchial valves (EBV) has become a guideline treatment in patients with advanced emphysema. Evidence from this minimally invasive treatment derives mainly from well-designed controlled trials conducted in high-volume specialized intervention centres. Little is known about real-life outcome data in hospitals setting up this novel treatment and which favourable conditions are required for a continuous successful program. Objectives: In this study, we aim to evaluate the eligibility rate for BLVR and whether the implementation of BLVR in our academic hospital is feasible and yields clinically significant outcomes. Method: A retrospec-
\end{abstract}

karger@karger.com www.karger.com/res

Karger!

GOPEN ACCESS
(C) 2021 The Author(s)

Published by S. Karger AG, Basel

This is an Open Access article licensed under the Creative Commons Attribution-NonCommercial-4.0 International License (CC BY-NC) (http://www.karger.com/Services/OpenAccessLicense), applicable to the online version of the article only. Usage and distribution for commercial purposes requires written permission. tive evaluation of patients treated with EBV between January 2016 and August 2019 was conducted. COPD assessment test $(\mathrm{CAT})$, forced expiratory volume in $1 \mathrm{~s}\left(\mathrm{FEV}_{1}\right)$, residual volume (RV), and 6-min walking test (6MWT) were measured at baseline and 3 months after intervention. Paired sample $t$ tests were performed to compare means before and after intervention. Results: Of 350 subjects screened, 283 (81\%) were not suitable for intervention mostly due to lack of a target lobe. The remaining 67 subjects (19\%) underwent bronchoscopic assessment, and if suitable, valves were placed in the same session. In total, 55 subjects (16\%) were treated with EBV of which 10 did not have complete follow-up: 6 subjects had their valves removed because of severe pneumothorax $(n=2)$ or lack of benefit $(n=4)$ and the remaining 4 had missing follow-up data. Finally, 45 patients had complete followup at 3 months and showed an average change \pm SD in CAT $-4 \pm 6$ points, $\mathrm{FEV}_{1}+190 \pm 140 \mathrm{~mL}$, RV $-770 \pm 790 \mathrm{~mL}$, and $+37 \pm 65 \mathrm{~m}$ on the $6 \mathrm{MWT}$ (all $p<0.001$ ). After 1 -year follow-
Correspondence to:

Rein Posthuma, reinposthuma@ciro-horn.nl 
up, 34 (76\%) subjects had their EBV in situ. Conclusion: Implementing BLVR with EBV is feasible and effective. Only $16 \%$ of screened patients were eligible, indicating that this intervention is only applicable in a small subset of highly selected subjects with advanced emphysema, and therefore a high volume of COPD patients is essential for a sustainable BLVR program.

(C) 2021 The Author(s)

Published by S. Karger AG, Basel

\section{Introduction}

Patients suffering from severe emphysema experience chronic dyspnoea and have impaired exercise tolerance and poor health-related quality of life. Despite optimal pharmacological therapy, smoking cessation, pulmonary rehabilitation (PR), and long-term oxygen therapy, the majority of the patients remain significantly disabled. Therefore, treatment is often focused on symptom relief and disease control [1].

Lung volume reduction surgery (LVRS) can provide clinical benefits in selected patients with predominantly upper lobe disease and low exercise capacity [2]. However, this procedure is associated with increased perioperative morbidity and mortality [3]. Similar to LVRS, endobronchial valve (EBV) treatment aims to reduce lung hyperinflation, by selectively targeting less functional areas of the lung with 1-way valves inducing atelectasis and is considered minimally invasive with lower rates of mortality and major postprocedural complications [4]. To date, there is increasing evidence that patients with advanced emphysema can experience meaningful improvements in lung function, dyspnoea, exercise capacity, physical activity, and quality of life after bronchoscopic lung volume reduction (BLVR) treatment with EBV [4-9], which were still present after 1-year followup $[10,11]$. The 2020 Report from the Global Initiative for Chronic Obstructive Lung Disease (GOLD) upgraded the level of evidence for EBV treatment to the highest evidence rating and supports the use of EBV treatment as standard care for select patients with advanced emphysema [1].

However, most evidence for the effectiveness of EBV treatment is available from large multi-centre randomized controlled trials conducted in centres with extensive experience in bronchoscopic interventions. Therefore, this study describes the patient selection, real-world clinical outcome data, and experiences in implementing EBV treatment in an academic hospital in the Netherlands with no prior experience in BLVR.

Implementation of Bronchoscopic Lung Volume Reduction
Table 1. Eligibility and ineligibility criteria for BLVR

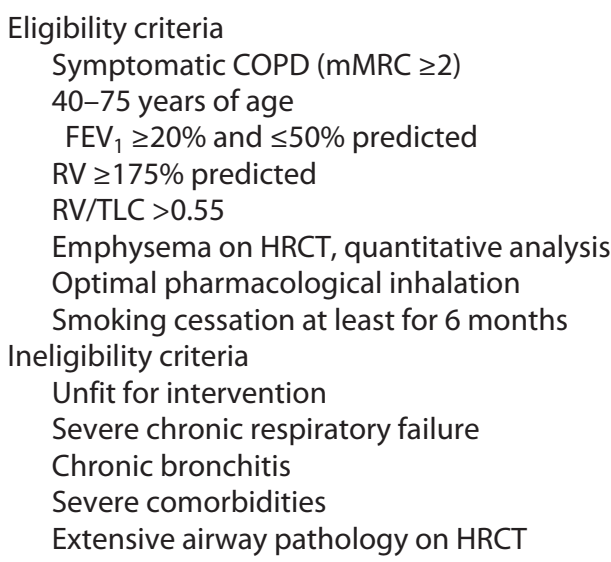

BLVR, bronchoscopic lung volume reduction; mMRC, Modified Medical Research Council Dyspnoea Scale; $\mathrm{FEV}_{1}$, forced expiratory volume in $1 \mathrm{~s}$; RV, residual volume; TLC, total lung capacity; HRCT, high-resolution computer tomography.

\section{Materials and Methods}

We performed a retrospective evaluation of all COPD patients screened for potential EBV treatment between January 2016, the start date of the BLVR programme, and August 2019 in the Maastricht University Medical Centre+, the Netherlands. Screened subjects were recruited from our own hospital clinic, local referral hospitals, and a tertiary PR clinic specialized in complex COPD affiliated with our hospital (CIRO, Horn, the Netherlands). Candidates underwent a stepwise evaluation from chart examination, extensive pulmonary function testing (PFT), and imaging to case discussion in a multidisciplinary team consisting of chest physicians, chest surgeon, and specialized chest radiologists, as patients became more eligible for BLVR.

Subjects were considered eligible for BLVR if they met the criteria (shown in Table 1) as were recommended in the expert recommendation [12]. The criteria were indicators for patient selection and not stringently applied, thus if a good target lobe was present in heterogeneous emphysema, a $\mathrm{FEV}_{1}$ below $20 \%$ was accepted. A high-resolution computed tomography was performed to assess the extent and distribution of emphysema and fissure completeness and to rule out contraindications for valve placement, like severe airway pathology or suspected nodules. A quantitative CT analysis (StratX@ software; PulmonX, Inc.) was performed to (1) quantify the severity of emphysema in each lobe by measuring the percentage of volume under a predefined density threshold of -910 Hounsfield units (HU) and below $-950 \mathrm{HU}[13$, 14], (2) quantify the lobar volume, and (3) examine the fissure integrity. In homogeneous emphysema, perfusion scintigraphy was performed, and if a target lobe showed clear diminished perfusion compared to the ipsilateral lobe, BLVR was considered.

Screening and consultation were done by dedicated chest physicians with experience in COPD and affinity with BLVR. Potential candidates were informed of the diagnostic results and treatment 


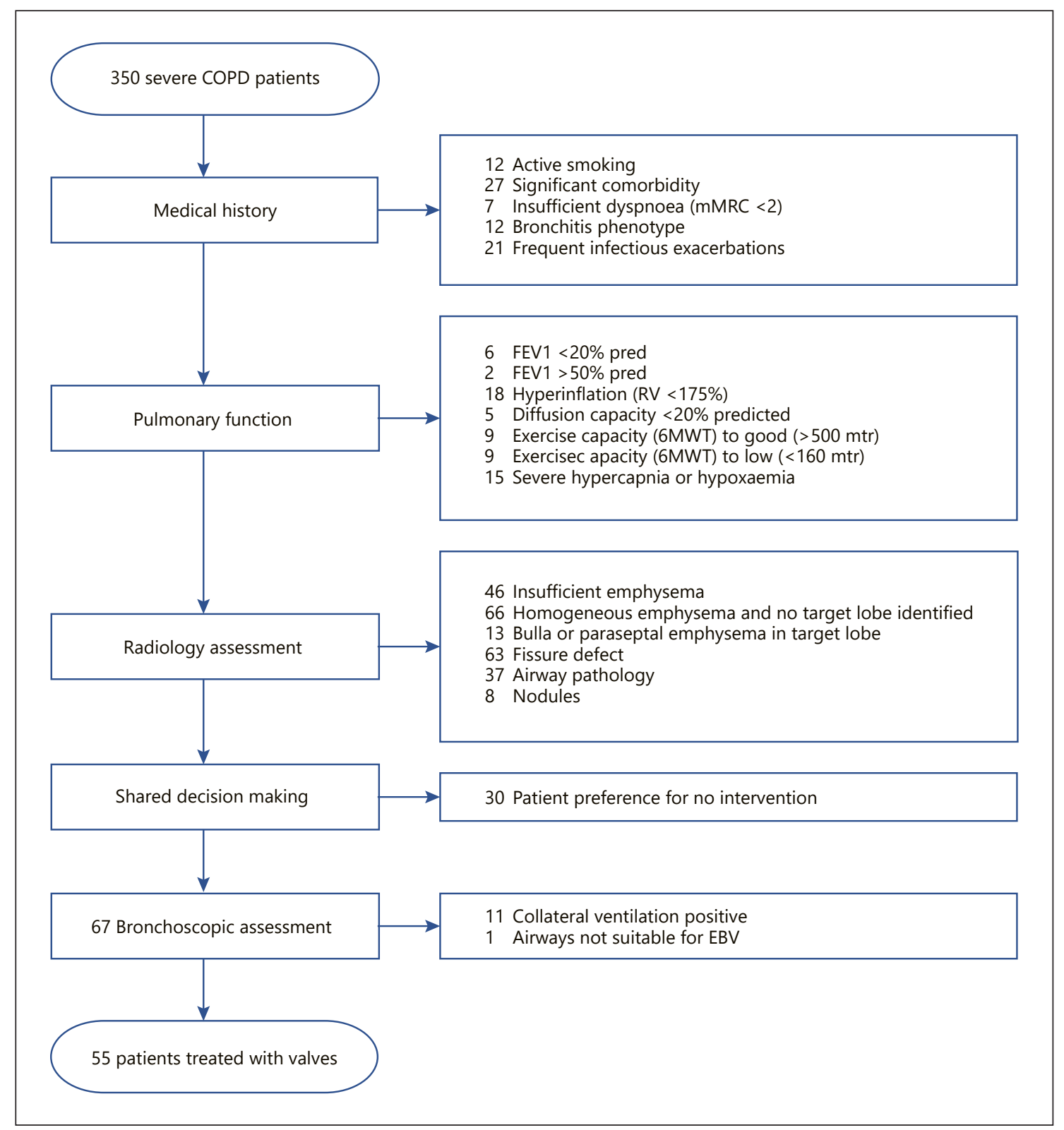

Fig. 1. Flowchart of screened COPD subjects and reason for exclusion. Data are shown as $n$. Counts reflect the number of subjects with the specific reason for ineligibility. Subjects could have multiple reasons for being unsuitable for BLVR. COPD, chronic ob-

advice given by the multidisciplinary team. Finally, shared decision-making between physician and candidate was the basis to perform BLVR. Prior to the intervention, subjects were stimulated to undergo an 8-week tertiary PR programme.

The intervention was performed under general anaesthesia in intubated subjects with mechanical ventilation support. Interlobar collateral ventilation was measured using the Chartis System ${ }^{\circledR}$ (Pulmonx Inc., Redwood City, CA, USA). Only Zephyr valves (PulmonX Inc., Redwood City, CA, USA) were used in our hospital. No antibiotic or corticosteroid prophylaxis was administered before structive pulmonary disease; BLVR, bronchoscopic lung volume reduction; EBV, endobronchial valve; $\mathrm{mMRC}$, Modified Medical Research Council Dyspnoea Scale; 6MWT, 6-Minute Walking Test; $\mathrm{m}$, metre; $\mathrm{FEV}_{1}$, forced expiratory volume in $1 \mathrm{~s}$.

BLVR. Treated subjects stayed admitted in the hospital for 5 days and were periodically followed up in our clinic after discharge.

Periodically, consultation was sought with a pulmonologist from the University Medical Centre Groningen, the Netherlands, for advice on BLVR. COPD assessment test (CAT), PFT including spirometry, body plethysmography, and 6-min walking test (6MWT) were performed at baseline and 3 months after the intervention. Outcomes were compared to minimal clinically import difference of 2 points on CAT score [15], $100 \mathrm{~mL}$ change in $\mathrm{FEV}_{1}$ [16], $25 \mathrm{~m}$ on 6MWT [17], and reduction of $430 \mathrm{~mL}$ in RV [18]. 
Table 2. Baseline characteristics of treated subjects $(n=45)$ who also completed 3-month follow-up

\begin{tabular}{|c|c|c|c|}
\hline Characteristics & Baseline & 3 months & $p$ value \\
\hline Age & $63.3 \pm 7.2$ & & \\
\hline Gender female, \% & 60 & & \\
\hline \multicolumn{4}{|l|}{ Lung function } \\
\hline $\mathrm{FEV}_{1}, \mathrm{~L}$ & $0.71 \pm 0.19$ & $0.90 \pm 0.23$ & $<0.001$ \\
\hline Response rate, \% & & 78 & \\
\hline $\mathrm{FEV}_{1}, \%$ predicted & $28 \pm 8$ & $35 \pm 10$ & $<0.001$ \\
\hline $\mathrm{TLC}, \mathrm{L}$ & $7.8 \pm 1.3$ & & \\
\hline TLC, $\%$ predicted & $138 \pm 14$ & & \\
\hline $\mathrm{RV}, \mathrm{L}$ & $5.2 \pm 1.0$ & $4.4 \pm 0.9$ & $<0.001$ \\
\hline Response rate, \% & & 67 & \\
\hline $\mathrm{RV}, \%$ predicted & $240 \pm 43$ & $204 \pm 38$ & $<0.001$ \\
\hline$D \mathrm{~L}_{\mathrm{CO}}, \%$ predicted & $37 \pm 9$ & & \\
\hline $6 \mathrm{MWD}, \mathrm{m}$ & $359 \pm 86$ & $396 \pm 88$ & $<0.001$ \\
\hline Response rate, \% & & 51 & \\
\hline 6MWD, \% predicted & $56 \pm 14$ & $62 \pm 13$ & $<0.001$ \\
\hline COPD assessment test $^{\dagger}$ & $21 \pm 5$ & $17 \pm 7$ & 0.001 \\
\hline Response rate, $\%$ & & 56 & \\
\hline \multicolumn{4}{|l|}{ Quantitative CT analysis $^{\ddagger}$} \\
\hline Target-lobe voxels below $-910 \mathrm{HU} \%$ & $69 \pm 8$ & & \\
\hline Target-lobe voxels below $-950 \mathrm{HU} \%$ & $44 \pm 11$ & & \\
\hline Target-lobe volume, $\mathrm{mL}$ & $1.790 \pm 452$ & & \\
\hline Prior pulmonary rehabilitation, $\%$ & 73 & & \\
\hline
\end{tabular}

Data are shown as mean or as percentage \pm SD and age in years. $L$, litre; TLC, total lung capacity; $\mathrm{RV}$, residual volume; $\mathrm{DL}$ co, diffusing capacity for carbon monoxide; $6 \mathrm{MWD}, 6$-minute walk distance; $\mathrm{HU}$, Hounsfield units; $\mathrm{mL}$, millilitre. Response rate = percentage of subjects reaching minimal clinically important difference. ${ }^{\dagger}$ Eight CAT scores were missing resulting in 37 pairs to compare. ${ }^{\ddagger}$ One quantitative $C T$ analysis was missing.
Data are presented as mean and standard deviation ( \pm ) unless noted otherwise. Outcomes were tested for normality with a Shapiro-Wilk test. Outcomes before and after intervention were compared with paired sample $t$ tests. The level of significance was set at $\leq 0.05$. Statistical analyses were performed using SPSS 25.0 (SPSS Inc., Chicago, IL, USA). The medical ethics committee judged that the Medical Research Involving Human Subjects Act (WMO) does not apply for this study since it consists of retrospective analyses with anonymous data (METC 2020-2328).

\section{Results}

During the study period, a total of 350 subjects with advanced COPD were screened for BLVR. Most subjects, 283 (81\%), had multiple characteristics that made them unsuitable (shown in Fig. 1). The most frequent contraindications were radiologic features, namely, homogeneous emphysema distribution and the absence of a target lobe in 66 cases (18.9\%). Second, PFT not meeting the inclusion criteria was an important contraindication in 64 subjects (18.2\%).

In a significant number of subjects $(n=30)$, shared decision-making between physician and candidate re- sulted in not preferring BLVR. Most of these subjects were fearful of potential complications, mainly pneumothorax. One subject had such a significant improvement in exercise capacity and symptoms after PR that inclusion criteria for BLVR no longer applied.

The remaining 67 subjects (19\%) underwent bronchoscopic assessment, and if suitable, valves were placed in the same session. In 12 subjects, no intervention was performed due to collateral ventilation $(n=11)$, or unexpected endobronchial lesion $(n=1)$ revealed during bronchoscopy. Fifty-five subjects (16\%) were treated with EBV. Ten did not have a complete follow-up at 3 months, including 6 subjects who had their valves removed because of severe pneumothorax $(n=2)$ or lacking treatment benefit $(n=4)$ and the remaining 4 subjects had missing data at follow-up. So finally, complete baseline and follow-up data were available in 45 subjects. The group had a mean age of 63 years with a mean $\mathrm{FEV}_{1}, \mathrm{RV}$, and $\mathrm{DLCO}_{\mathrm{sb}}$ of, respectively, $28 \%, 240 \%$, and $37 \%$ of predicted. Baseline and follow-up values are presented in Table 2. The mean changes in outcomes are shown in Figure 2. 


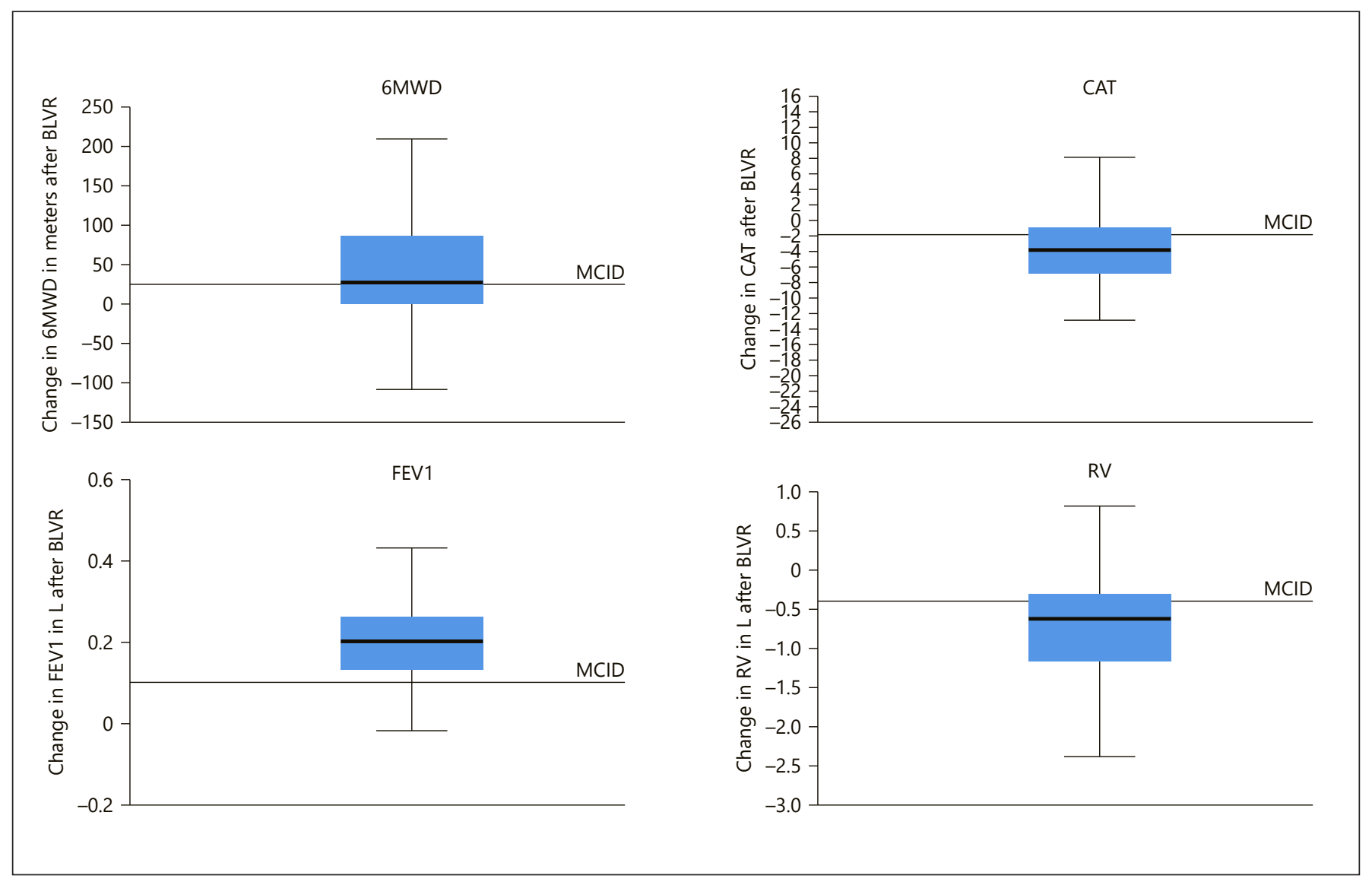

Fig. 2. Outcomes at 3 months. Shown are the changes in outcome at 3 months in subjects treated with EBV. Black horizontal lines represent the MCID of 2 points in CAT score, $100 \mathrm{~mL}$ change in $\mathrm{FEV}_{1}, 25 \mathrm{~m}$ on $6 \mathrm{MWT}$, and a reduction of $430 \mathrm{~mL}$ in RV. EBV,

Table 3. Postprocedural complications in the 55 subjects treated with BLVR during the first 3 months

\begin{tabular}{ll}
\hline Complication & $N=55$ \\
\hline Pneumothorax & $5(9.1)$ \\
Postprocedure COPD exacerbation & $15(27.3)$ \\
Haemoptysis & $2(3.6)$ \\
Torsion of the bronchus & $2(3.6)$ \\
Pneumonia or airway infection requiring antibiotics & $17(30.9)$ \\
Valve expectoration & $1(1.8)$ \\
Temporarily $\mathrm{O}_{2}$ supplementation & $9(16.4)$ \\
Mortality at 3 months & $0(0)$ \\
\hline
\end{tabular}

Data are shown as $n$ (\%). Counts reflect number of subjects reporting the specific complication. Subjects could have multiple complications. BLVR, bronchoscopic lung volume reduction; COPD, chronic obstructive pulmonary disease. endobronchial valve; MCID, minimal clinically important difference; $\mathrm{FEV}_{1}$, forced expiratory volume in $1 \mathrm{~s} ; 6 \mathrm{MWD}, 6$-min walk distance; RV, residual volume; CAT, COPD assessment test.

Thirty-three (73\%) of the $45 \mathrm{BLVR}$ patients underwent an 8 -week internal PR at CIRO within 1 year before the intervention. There was no difference in outcome between the subjects who did or did not undergo PR before BLVR.

After 1-year follow-up, 34 of the 45 (76\%) treated subjects had their EBV in situ. The most prominent reason for removal of EBV was loss of benefit mostly due to granulation tissue $(n=5)$. Other reasons for removal were recurrent pneumonia $(n=2)$, torsion of the bronchus $(n=1)$, lack of benefit $(n=1)$, and diffuse endobronchial bronchitis reaction $(n=1)$. One patient died within 1 year after BLVR (not-BLVR related, acute intestinal bleeding). Of the 34 patients with EBV in situ at 1 year, 5 did not have a follow-up visit mostly due to not attending the visit, resulting in 29 patients with 1 -year pulmonary function data; in 2 patients, RV was not performed, and in 7 patients, the 6MWD was missing. After 
1 year, the mean change in $\mathrm{FEV}_{1}$ was $+101 \pm 176 \mathrm{~mL}$ ( $p$ $<0.05), \mathrm{RV}-527 \pm 842 \mathrm{~mL}(p<0.05)$, and $6 \mathrm{MWD}+7 \pm$ $55 \mathrm{~m}(p>0.05)$.

The most common adverse events, represented in Table 3, were airway infections requiring antibiotics and post-intervention COPD exacerbations. Slightly over half of the subjects (54.5\%) experienced at least 1 complication of which most were easily manageable and not severe. No mortality occurred in the first 3 months after intervention. The pneumothorax rate in our cohort was $9 \%$, and all were treated with a pleural chest catheter. If necessary, single or complete valve removal was performed to allow expansion of the collapsed lung, which was the case in 6 subjects. Nine subjects required temporarily oxygen supplementation during hospitalization or after discharge but could be stopped before the first follow-up visit and was mostly attributed to COPD exacerbation.

\section{Discussion}

We report our experience with the implementation and results of BLVR using 1-way valves in an academic hospital. Only $16 \%$ of the severe COPD subjects in our cohort were eligible for EBV, indicating that this intervention is only applicable in a small subset of highly selected subjects with advanced emphysema. Therefore, a high volume of COPD patients is essential for a sustainable BLVR program.

Furthermore, outcomes at 3 months in real-life setting indicate that implementation is feasible and safe and that treated subjects demonstrate clinical meaningful improvements. At 12 months, the results are less pronounced.

Strict patient selection is crucial in achieving beneficial results and keeping the postprocedural morbidity and mortality rate low. Some literature exists on studying reasons for not receiving interventional treatment in severe COPD patients in real-life setting. Our results indicate that radiological features such as lack of or homogeneous emphysema and the inability to identify a good target lobe were the main reasons of denying treatment, followed by insufficient hyperinflation and unsuitable clinical condition. This is in line with previous reports; however, eligibility proportions are variable. May et al. [19] described in a single-centre retrospective cohort of 138 potential candidates an eligibility rate of $27.5 \%$, though different BLVR techniques (EBV, coils, and vapour) were used, therefore probably

Implementation of Bronchoscopic Lung

Volume Reduction causing a higher acceptance for intervention when compared to EBV alone. A recent study by Welling et al. [20] reported a selection rate of $19 \%$ out of 1,500 referrals for BLVR, but also different interventions were available. The most common reported contraindication in that study was lack of appropriate target lobe and unsuitable COPD phenotype. A specialized pulmonary intervention centre in Germany evaluated the reason for not performing lung volume reduction in 115 patients and reported incomplete fissures and absence of proper target lobe as the main contraindications with an eligibility rate of $14 \%$ [21].

Interestingly, in our centre, a significant proportion of patients $(8.6 \%)$ were not treated based on shared decision-making between chest physician and patient, when advantages and disadvantages were discussed in detail. Some of these patients were fearful of potential complications. Additionally, screening for BLVR in COPD patients not specifically referred for this treatment, as was done during the baseline assessment for $\mathrm{PR}$, could also be an explanation. It is reasonable to assume that most patients referred to a centre for BLVR already decided that, if eligible, are willing to undergo BLVR. This in contrary to when BLVR is one of the potential options in an assessment specified for PR. Shared decision-making has not been addressed as a major reason for non-treatment in previous studies.

The benefits from EBV treatment in this report are, in general, consistent with other real-world efficacy studies $[11,22]$. However, treatment results vary due to differences in inclusion criteria, primary outcome, and followup duration.

Skowasch et al. [22] reported in a prospective, observational, single-arm multi-centre trial in Germany at 6-month follow-up a mean improvement of $+100 \mathrm{~mL}$ in $\mathrm{FEV}_{1}$ and an $\mathrm{RV}$ reduction of $-0.42 \mathrm{~L}$ which are just around the minimal clinically import difference cutoff values. Gompelmann et al. [11] reported in a retrospective cohort with a follow-up period up to 3 years efficacy with gradual decline of the lung function parameters over time. This decline is also demonstrated in our report and could be due to progression of COPD or loss of benefit of the EBV. Randomized controlled trials showed in general more pronounced outcomes [4, 6, 7]. Klooster et al. [6] reported in the STELVIO trial, a single-centre randomized controlled study, an average increase in $\mathrm{FEV}_{1}$ of $161 \mathrm{~mL}$ and 6MWD of $60 \mathrm{~m}$ at 6 months in the intentionto-treat population. Criner et al. [4] reported in a large multi-centre trial in patients with heterogeneous emphysema, sustainable outcomes after 12 months of follow-up. 
During the implementation of our BLVR, we were reserved about treating patients with homogeneous emphysema, since outcomes of homogeneous patients were not well reported, and benefit was mainly described for patients with a heterogeneous distribution of emphysema. This approach changed in some degree with the IMPACT study published late 2016 [7]. The trial reported positive 3-month outcome of EBV therapy in patients with homogeneous emphysema, although the effects appeared smaller in comparison with heterogeneous emphysema-treated patients.

The most common complication after BLVR in our cohort was airway infection requiring antibiotics. The cause of this high prevalence is not known and could be due to not administering prophylactic antibiotics. Another explanation could be the careful approach in the startup phase, when antibiotics were sometimes started out of caution when patients reported increased sputum or cough after the intervention. The pneumothorax incidence was $9.3 \%$, which is at the lower end of reported rates in the different trials (8-34\%) [4-8]. Pneumothorax can also be used as a surrogate marker for achieving atelectasis, since the complication is mostly due to overexpansion of the ipsilateral untreated lobe. Pneumothorax was treated according to expert statement by Valipour et al. [23] and mostly required only chest tube drainage. Furthermore, new insights into the risk factors for pneumothorax became available [24] during our start-up phase, including para-septal emphysema, pleura-adhesion, or extreme large volume of the target lobe. These features are now mostly avoided. Since severe pneumothorax with persistent air leakage could need surgical intervention, in-hospital thoracic surgery facilities are advisory. Overall, it has been reported that the complication rate is higher in treated patients compared to the standard of care group, but only directly in the first 45 days after treatment; after this period, the frequency of complications was comparable between groups, even a tendency to fewer exacerbations, but this did not reach statistical significance [4].

A limitation of this study is that it is single centre, retrospective, and has limited length of complete follow-up. Also, not including the lost to follow-up subjects in the analysis gives a slightly overestimation of the reported outcomes.

Additionally, there is a lack of comparison with a control group. However, the strength is its real-life background without a strictly controlled research setting, which could make it more extrapolatable to the everyday practice.
Here, we discuss the lessons learned during our implementation process and the conditions necessary for a durable BLVR program. First, as many patients need to be screened and discussed for potential treatment, a dedicated COPD clinic seems to be important with anchoring as a regional referral centre maintaining a certain flow of patients to build up and maintain expertise. When starting up a BLVR program, a clear protocol of the patient selection, the intervention, and the clinical follow-up is key for self-evaluation of achieved results. A multidisciplinary approach and discussion, including the managing physician, the interventionist, and a committed chest radiologist, is needed. In addition, close contact with a high-volume experienced treatment centre to discuss the eligibility of more complex cases and complication management has been very helpful during our implementation phase. Availability of thoracic surgery is important for complication management but also for integration with an LVRS program. With more dedicated emphysema imaging, more subjects eligible for lung volume surgery are identified. In addition, subjects with late lobe reinflation after valve treatment because of, e.g., granulation tissue and with an initial good response might be good candidates for a surgical intervention [25]. Finally, a good alliance with a PR centre including a thorough assessment and integrated personalized program is recommended to optimize the patients before the intervention. However, if PR before BLVR is the best sequence or better results can be achieved when PR is planned after the intervention is not known and is currently being investigatedin theSoLVE-trial(clinicaltrials.govNCT03474471).

In conclusion, implementing BLVR with EBV is feasible, safe, and effective in start-up centres. This intervention is only applicable in a small subset of highly selected patients with advanced emphysema, indicating that a large flow of COPD patients is necessary for a sustainable program.

\section{Acknowledgments}

We would like to thank Dr. K. Klooster and Prof. Dr. D.J. Slebos as they provided their time and advice during the start-up phase of our program.

\section{Statement of Ethics}

This study is exempt from ethics committee approval. The medical ethics committee (METC azM/UM) judged that the Medical Research Involving Human Subjects Act (WMO) does not ap- 
ply for this study since it consists of retrospective analyses with anonymous data (METC 2020-2328). Official approval from the METC aZM/UM is not required.

\section{Conflict of Interest Statement}

R.P. has received honoraria for lectures from Health investment, Medtalks, and Chronisch Zorgnet, A.W.V. has no conflicts of interest, K.H.M.W. has no conflicts of interest, P.S.N. has no conflicts of interest, J.U.S. has no conflicts of interest, H.A.G. has no conflicts of interest, G.W. has no conflicts of interest, E.F.M.W. has no conflicts of interest, L.E.G.W.V. reports consulting fees from GSK and AstraZeneca, honoraria for lectures from GSK, AstraZeneca, Resmed, and Boehringer, and the Data Safety Monitoring Board, AstraZeneca.

\section{Funding Sources}

This study was supported by the Lung Foundation Netherlands grant: 5.1.17.171.0.

\section{Author Contributions}

Posthuma, Wouters, and Vanfleteren contributed to conception and design. Posthuma, Vaes, Vanfleteren, and Gietema contributed to data acquisition and analysis. All authors contributed to data interpretation, manuscript writing, and final approval of the manuscript and agree to be accountable for all aspects of the work.

\section{Data Availability Statement}

All data generated or analysed during this study are included in this article. Further enquiries can be directed to the corresponding author.

\section{References}

1 The Global Initiative for Chronic Obstructive Lung Disease (GOLD). Global strategy for the diagnosis, management and prevention of chronic obstructive pulmonary disease; 2020. Available from: https://goldcopd.org/goldreports/.

2 Geddes D, Davies M, Koyama H, Hansell D, Pastorino U, Pepper J, et al. Effect of lungvolume-reduction surgery in patients with severe emphysema. N Engl J Med. 2000 Jul 27; 343(4):239-45.

3 Decker MR, Leverson GE, Jaoude WA, Maloney JD. Lung volume reduction surgery since the national emphysema treatment trial: study of society of thoracic surgeons database. J Thorac Cardiovasc Surg. 2014 Dec;148(6): 2651-e1.

4 Criner GJ, Sue R, Wright S, Dransfield M, Rivas-Perez $\mathrm{H}$, Wiese T, et al. A multicenter randomized controlled trial of zephyr endobronchial valve treatment in heterogeneous emphysema (LIBERATE). Am J Respir Crit Care Med. 2018 Nov 1;198(9):1151-64.

5 Davey C, Zoumot Z, Jordan S, McNulty WH, Carr DH, Hind MD, et al. Bronchoscopic lung volume reduction with endobronchial valves for patients with heterogeneous emphysema and intact interlobar fissures (the BeLieVeRHIFi study): a randomised controlled trial. Lancet. 2015 Sep 12;386(9998):1066-73.

6 Klooster K, ten Hacken NH, Hartman JE, Kerstjens HA, van Rikxoort EM, Slebos DJ. Endobronchial valves for emphysema without interlobar collateral ventilation. N Engl J Med. 2015 Dec 10;373(24):2325-35.

7 Valipour A, Slebos DJ, Herth F, Darwiche K, Wagner M, Ficker JH, et al. Endobronchial valve therapy in patients with homogeneous emphysema. Results from the IMPACT study. Am J Respir Crit Care Med. 2016 Nov 1;194(9):1073-82.

8 Kemp SV, Slebos DJ, Kirk A, Kornaszewska M, Carron K, Ek L, et al. A multicenter randomized controlled trial of zephyr endobronchial valve treatment in heterogeneous emphysema (TRANSFORM). Am J Respir Crit Care Med. 2017 Dec 15;196(12):1535-43.

9 Criner GJ, Delage A, Voelker K, Hogarth DK, Majid A, Zgoda M, et al. Improving lung function in severe heterogenous emphysema with the spiration valve system (EMPROVE). A multicenter, open-label randomized controlled clinical trial. Am J Respir Crit Care Med. 2019 Dec 1;200(11):1354-62.

10 Klooster K, Hartman JE, Ten Hacken NH, Slebos DJ. One-year follow-up after endobronchial valve treatment in patients with emphysema without collateral ventilation treated in the STELVIO trial. Respiration. 2017;93(2):112-21.

11 Gompelmann D, Heinhold T, Rotting M, Bischoff E, Kontogianni K, Eberhardt R, et al. Long-term follow up after endoscopic valve therapy in patients with severe emphysema. Ther Adv Respir Dis. 2019 Jan; 13: 1753466619866101.

12 Slebos DJ, Shah PL, Herth FJ, Valipour A. Endobronchial valves for endoscopic lung volume reduction: best practice recommendations from expert panel on endoscopic lung volume reduction. Respiration. 2017;93(2): 138-50.

13 Muller NL, Staples CA, Miller RR, Abboud RT. "Density mask". An objective method to quantitate emphysema using computed tomography. Chest. 1988 Oct;94(4):782-7.

14 Gevenois PA, de Maertelaer V, De Vuyst P, Zanen J, Yernault JC. Comparison of computed density and macroscopic morphometry in pulmonary emphysema. Am J Respir Crit Care Med. 1995 Aug;152(2): 653-7.

15 Kon SS, Canavan JL, Jones SE, Nolan CM, Clark AL, Dickson MJ, et al. Minimum clinically important difference for the COPD assessment test: a prospective analysis. Lancet Respir Med. 2014 Mar;2(3):195-203.

16 Donohue JF. Minimal clinically important differences in COPD lung function. COPD. 2005 Mar;2(1):111-24.

17 Holland AE, Hill CJ, Rasekaba T, Lee A, Naughton MT, McDonald CF. Updating the minimal important difference for six-minute walk distance in patients with chronic obstructive pulmonary disease. Arch Phys Med Rehabil. 2010 Feb;91(2):221-5.

18 Hartman JE, Ten Hacken NH, Klooster K, Boezen HM, de Greef MH, Slebos DJ. The minimal important difference for residual volume in patients with severe emphysema. Eur Respir J. 2012 Nov;40(5):1137-41.

19 May N, Niehaus-Gebele C, Reichenberger F, Behr J, Gesierich W. Screening for bronchoscopic lung volume reduction: reasons for not receiving interventional treatment. Lung. 2020 Feb;198(1):221-8.

20 Welling JBA, Hartman JE, Augustijn SWS, Kerstjens HAM, Vanfleteren L, Klooster K, et al. Patient selection for bronchoscopic lung volume reduction. Int J Chron Obstruct Pulmon Dis. 2020;15:871-81.
Implementation of Bronchoscopic Lung Volume Reduction 
21 Polke M, Rotting M, Sarmand N, Krisam J, Eberhardt R, Herth FJF, et al. Interventional therapy in patients with severe emphysema: evaluation of contraindications and their incidence. Ther Adv Respir Dis. 2019 Jan-Dec; 13:1753466619835494.

22 Skowasch D, Fertl A, Schwick B, Schafer H, Hellmann A, Herth FJ, et al. A long-term follow-up investigation of endobronchial valves in emphysema (the LIVE study): study proto- col and six-month interim analysis results of a prospective five-year observational study. Respiration. 2016;92(2):118-26.

23 Valipour A, Slebos DJ, de Oliveira HG, Eberhardt R, Freitag L, Criner GJ, et al. Expert statement: pneumothorax associated with endoscopic valve therapy for emphysema: potential mechanisms, treatment algorithm, and case examples. Respiration. 2014;87(6):51321.
24 van Geffen WH, Klooster K, Hartman JE, Ten Hacken NHT, Kerstjens HAM, Wolf RFE, et al. Pleural adhesion assessment as a predictor for pneumothorax after endobronchial valve treatment. Respiration. 2017;94(2):224-31.

25 Caviezel C, Guglielmetti LC, Ladan M, Hansen HJ, Perch M, Schneiter D, et al. Lung volume reduction surgery as salvage procedure after previous use of endobronchial valves. Interact Cardiovasc Thorac Surg. 2020 Dec 6. 